\title{
Ajout d'équivalents des groupes alimentaires au Questionnaire canadien de fréquence alimentaire II pour estimer l'Indice canadien de saine alimentation-2005
}

\author{
Maria McInerney, M. Sc. (1); Vikki Ho, Ph. D. (2); Anita Koushik, Ph. D. (2); Isabelle Massarelli, nutritionniste (3); \\ Isabelle Rondeau, nutritionniste (3); Gavin R. McCormack, Ph. D. (1); Ilona Csizmadi, Ph. D. (1)
}

Cet article de recherche quantitative originale a fait l'objet d'une évaluation par les pairs.

\section{Résumé}

Introduction : Il a été prouvé qu’un régime alimentaire de piètre qualité augmente le risque de maladies chroniques courantes susceptibles de nuire à la qualité de vie et d'alourdir le fardeau qui pèse sur le système de santé. Les recommandations fondées sur des données probantes du Guide alimentaire canadien (GAC) fournissent des conseils nutritionnels destinés à améliorer la qualité du régime alimentaire. L'Indice canadien de saine alimentation (ICSA), un outil de mesure de la qualité du régime alimentaire, permet d'évaluer la conformité au GAC. Le Questionnaire canadien de fréquence alimentaire II (QFA-C II) [Canadian Diet History Questionnaire II, C-DHQ II], mis au point récemment, pourrait quant à lui servir à estimer l'ICSA au sein de la population canadienne si on pouvait ajouter à sa base de données sur les éléments nutritifs les équivalents des groupes alimentaires (correspondant aux portions du GAC). Nous décrivons dans cet article des méthodes destinées à enrichir cette base de données sur les éléments nutritifs du QFA-C II en vue d'estimer l'ICSA.

Méthodologie : Nous avons créé des équivalents des groupes alimentaires à partir de données provenant de diverses bases de données sur les aliments et les éléments nutritifs, en particulier l'Enquête sur la santé dans les collectivités canadiennes, cycle 2.2 Nutrition de 2004. Nous avons ajouté ces variables à la base de données sur les éléments nutritifs du QFA-C II. Nous avons déterminé les scores de l'ICSA et avons effectué des analyses descriptives pour les participants qui ont répondu au QFA-C II dans le cadre d'une étude transversale canadienne.

Résultats : Le score moyen de l'ICSA dans notre échantillon de 446 adultes de 20 à 83 ans était de 64,4 (écart-type : 10,8). Les femmes, les non-fumeurs et les personnes ayant un niveau de scolarité supérieur au secondaire ont obtenu de manière statistiquement significative des notes plus élevées que les hommes, les fumeurs et les personnes détenant un diplôme d'études secondaires ou moins.

Conclusion : La possibilité d'évaluer l'ICSA à l'aide du QFA-C II facilite l'étude de la qualité du régime alimentaire et de l'état de santé de la population canadienne.

Mots-clés : qualité du régime alimentaire, indice de saine alimentation, questionnaire de fréquences alimentaires, évaluation nutritionnelle, base de données sur les éléments nutritifs

\section{Introduction}

Les indices alimentaires sont des mesures multidimensionnelles fondées sur plusieurs composantes du régime alimentaire et aptes à évaluer la qualité du régime alimentaire ${ }^{1}$. L'élaboration, l'évaluation et l'application des indices alimentaires suscitent un intérêt croissant en raison du lien entre régime alimentaire, maladies chroniques ${ }^{2-5}$ et risque de mortalité ${ }^{6}$. En matière de surveillance, certains indices alimentaires sont utiles pour déterminer dans quelle mesure les populations respectent les recommandations nutritionnelles nationales ${ }^{7,8}$.

Le Healthy Eating Index (HEI) ${ }^{9}$, un outil de mesure de la qualité du régime alimentaire,
Points saillants

- Un régime alimentaire de grande qualité est important pour prévenir les maladies chroniques.

- On peut utiliser l'Indice canadien de saine alimentation (ICSA), une échelle de notation du type d'alimentation reposant sur les recommandations nutritionnelles en fonction de l'âge et du sexe de l'édition de 2007 de Bien manger avec le Guide alimentaire canadien (GAC), pour mesurer et surveiller la qualité du régime alimentaire.

- Pour calculer l'ICSA, on doit disposer des équivalents des groupes alimentaires (correspondant aux portions du GAC), mais ces variables sont absentes de la plupart des bases de données canadiennes sur les éléments nutritifs.

- Nous avons suivi une démarche rigoureuse et valide pour ajouter des équivalents des groupes alimentaires à la base de données sur les éléments nutritifs du Questionnaire canadien de fréquence alimentaire II (QFA-C II).

- Il est maintenant possible d'utiliser le QFA-C II pour évaluer l'ICSA et ainsi déterminer la qualité du régime alimentaire de la population canadienne.

a été élaboré initialement par le département de l'Agriculture des États-Unis. Les similitudes entre les recommandations nutritionnelles du Canada et celles des États-Unis facilitent l'adaptation du HEI au contexte canadien ${ }^{8,10-13}$. L'Indice canadien de saine alimentation (ICSA) 2005 est ainsi

Rattachement des auteurs :

1. Département des sciences de la santé communautaire, Cumming School of Medicine, Université de Calgary, Calgary (Alberta), Canada

2. CRCHUM (Centre de recherche du CHUM) et Département de médecine sociale et préventive, Université de Montréal, Montréal (Québec), Canada

3. Bureau de la surveillance des aliments et de l'intégration de la science, Direction des aliments, Santé Canada, Ottawa (Ontario), Canada

Correspondance : Maria McInerney, Département des sciences de la santé communautaire, Cumming School of Medicine, Université de Calgary, 3280, Hospital Drive NW, Calgary (Alberta) T2N 4Z6; courriel : mcmciner@ucalgary.ca 
le fruit d'une démarche d'adaptation ${ }^{8}$ et s'appuie sur les recommandations et les équivalences de portion de l'édition de 2007 de Bien manger avec le Guide alimentaire canadien (GAC) ${ }^{14}$. Par conséquent, il intègre les recommandations nutritionnelles canadiennes en fonction de l'âge et du sexe et permet également de surveiller et d'évaluer la qualité du régime alimentaire des Canadiens ${ }^{8,10}$.

On mesure l'ICSA sur une échelle de 0 à 100 afin de rendre compte de la qualité globale de l'alimentation à partir des deux composantes que sont la suffisance et la modération $^{8}$, les scores plus élevés correspondant à un meilleur respect des recommandations du GAC. La composante " suffisance » de l'ICSA rend compte de la consommation totale de fruits et de légumes, de fruits entiers, de légumes vert foncé et orangés, de lait et de substituts laitiers, de viande et de substituts de viande, de produits céréaliers, de produits à grains entiers et de lipides insaturés. La composante " modération » rend compte des apports de sodium, de lipides saturés et d'autres aliments non recommandés dans le GAC. Le tableau 1 présente les critères de calcul des scores de l'ICSA ${ }^{8}$.

Le Questionnaire canadien de fréquence alimentaire II (QFA-C II) [Canadian Diet History Questionnaire II, C-DHQ II], un questionnaire de fréquence alimentaire global, constitue une adaptation à usage canadien du DHQ II du National Cancer Institute des États-Unis. Ont été utilisées les données sur l'apport alimentaire tirées de l'Enquête sur la santé dans les collectivités canadiennes, cycle 2.2 Nutrition de $2004^{15}$ pour la création des 331 catégories d'aliments de sa base de données. Le QFA-C II, disponible en ligne ${ }^{16,17}$, est employé dans de nombreuses études canadiennes actuelles $^{18,19}$. On envisage de plus en plus d'y recourir pour évaluer la qualité du régime alimentaire, comme ce fut le cas d'une version antérieure du questionnaire ${ }^{20}$.

Nous décrivons ici les méthodes employées afin de créer pour le QFA-C II les équivalents des groupes alimentaires (correspondant aux portions du GAC) nécessaires au calcul de l'ICSA (consommation totale de fruits et légumes, fruits entiers, légumes vert foncé et orangés, lait et substituts, viande et substituts, produits céréaliers, grains entiers, et autres aliments). Nous présentons également les équivalents de

TABLEAU 1

Critères d'évaluation de l'Indice canadien de saine alimentation (ICSA)

\begin{tabular}{|c|c|c|}
\hline $\begin{array}{l}\text { Composante } \\
\text { (groupe alimentaire) }\end{array}$ & $\begin{array}{l}\text { Fourchette } \\
\text { de scores }\end{array}$ & Critères d'attribution des scores \\
\hline Suffisance $^{\mathrm{a}}$ & 0 à 60 points & \\
\hline \multirow{2}{*}{$\begin{array}{l}\text { Consommation totale } \\
\text { de légumes et de fruits }\end{array}$} & \multirow{2}{*}{0 à 10 points } & Minimum : 0 \\
\hline & & Maximum : 4 à 10 portions ${ }^{b}$ \\
\hline \multirow[b]{2}{*}{ Fruits entiers } & \multirow[b]{2}{*}{0 à 5 points } & Minimum : 0 \\
\hline & & $\begin{array}{l}\text { Maximum : } 0,8 \text { à } 2,1 \text { portions ( } 21 \% \text { de la consomma- } \\
\text { tion totale recommandée de légumes et fruits) }{ }^{b}\end{array}$ \\
\hline \multirow{2}{*}{$\begin{array}{l}\text { Légumes vert foncé } \\
\text { et orangés }\end{array}$} & \multirow[b]{2}{*}{0 à 5 points } & Minimum : 0 \\
\hline & & $\begin{array}{l}\text { Maximum : } 0,8 \text { à } 2,1 \text { portions ( } 21 \% \text { de la consomma- } \\
\text { tion totale recommandée de légumes et fruits) }{ }^{b}\end{array}$ \\
\hline \multirow{2}{*}{$\begin{array}{l}\text { Consommation totale } \\
\text { de produits céréaliers }\end{array}$} & \multirow{2}{*}{0 à 5 points } & Minimum : 0 \\
\hline & & Maximum : 3 à 8 portions ${ }^{b}$ \\
\hline \multirow[b]{2}{*}{ Produits à grains entiers } & \multirow[b]{2}{*}{0 à 5 points } & Minimum : 0 \\
\hline & & $\begin{array}{l}\text { Maximum : } 1,5 \text { à } 4 \text { portions ( } 50 \% \text { de la consomma- } \\
\text { tion totale recommandée de produits céréaliers) }{ }^{\mathrm{b}}\end{array}$ \\
\hline \multirow{2}{*}{ Lait et substituts } & \multirow{2}{*}{0 à 10 points } & Minimum : 0 \\
\hline & & Maximum : 2 à 4 portions ${ }^{b}$ \\
\hline \multirow{2}{*}{ Viandes et substituts } & \multirow{2}{*}{0 à 10 points } & Minimum : 0 \\
\hline & & Maximum : 1 à 3 portions (75 à 225 grammes) $^{b}$ \\
\hline \multirow{2}{*}{ Lipides insaturés } & \multirow{2}{*}{0 à 10 points } & Minimum : 0 \\
\hline & & Maximum : 30 à 45 grammes $^{b}$ \\
\hline Modérationc $^{c}$ & 0 à 40 points & \\
\hline \multirow{2}{*}{ Lipides saturés } & 8 à 10 points & \multirow{2}{*}{ Maximum : 7 à $10 \%$ de l'apport énergétique total } \\
\hline & 0 à 8 points & \\
\hline \multirow{2}{*}{ Sodium } & 8 à 10 points & \multirow{2}{*}{ Apport suffisant à apport maximal tolérable } \\
\hline & 0 à 8 points & \\
\hline \multirow{2}{*}{ «Autres aliments » } & \multirow{2}{*}{0 à 20 points } & Minimum : 5 \% ou moins de l'apport énergétique total \\
\hline & & Maximum : $40 \%$ ou plus de l'apport énergétique total \\
\hline
\end{tabular}

Source : Garriguet D, La qualité de l'alimentation au Canada. Rapports sur la santé. 2009;20(3):41-52.

a Pour les composantes de la suffisance, 0 point pour une valeur égale ou inférieure au minimum, 5 ou 10 points pour une valeur égale ou supérieure au maximum, et nombre proportionnel de points pour les quantités entre le minimum et le maximum.

${ }^{\text {b }}$ Selon l'âge et le sexe, conformément au Guide alimentaire canadien.

c Pour les composantes de la modération, 10 ou 20 points pour une valeur égale ou inférieure au minimum, 0 point pour une valeur égale ou supérieure au maximum, et nombre proportionnel de points pour les quantités entre le minimum et le maximum.

groupes alimentaires et les scores de l'ICSA calculés à partir du QFA-C II pour un échantillon d'adultes canadiens participant à l'étude Pathways to Health Study ${ }^{21}$. Cet article est le fruit d'une étude plus vaste décrite dans le mémoire de maîtrise Associations between the Neighbourhood Food Environment, Neighbourhood Socioeconomic Status and Diet Quality in Canadian Adults ${ }^{22}$.

\section{Méthodologie}

La figure 1 offre un aperçu du processus employé pour obtenir l'ICSA à partir du
QFA-C II. Huit nouvelles variables correspondant aux équivalents de groupes alimentaires (portions du GAC) pour la consommation totale de fruits et légumes, les fruits entiers, les légumes vert foncé et orangés, le lait et ses substituts, la viande et ses substituts, les produits céréaliers, les grains entiers, et les autres aliments ont été créées pour chacune des 331 catégories d'aliments de la base de données sur les éléments nutritifs du QFA-C II. Les algorithmes utilisés pour créer les nouvelles variables à partir des aliments de l'Enquête sur la santé dans les collectivités canadiennes sont présentés figure 2 . 
FIGURE 1

Aperçu du processus

331 catégories d'aliments de la base de données sur les éléments nutritifs du QFA-C II

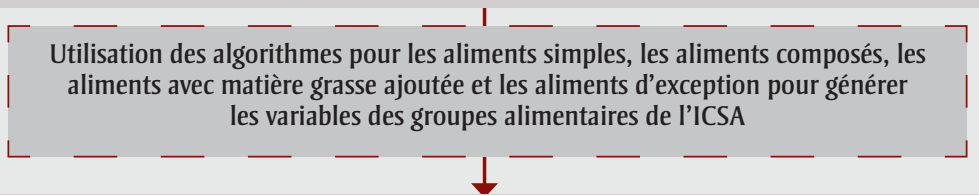

Intégration de 8 nouvelles variables d'équivalents des groupes alimentaires de l'ICSA à la base de données sur les éléments nutritifs du QFA-C II :

- Équivalents des groupes alimentaires pour les légumes et les fruits

- Équivalents des groupes alimentaires pour les fruits entiers

- Équivalents des groupes alimentaires pour les légumes vert foncé et orangés

- Équivalents des groupes alimentaires pour la consommation totale de produits céréaliers

- Équivalents des groupes alimentaires pour les grains entiers

- Équivalents des groupes alimentaires pour le lait et les substituts

- Équivalents des groupes alimentaires pour la viande et les substituts

- Nombre total de calories provenant d'« autres aliments » non recommandés dans le GAC

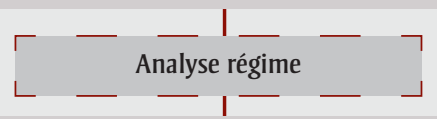

Extrant QFA-C II : apport quotidien des équivalents des groupes alimentaires

Macro SAS pour générer le score de I'ICSA

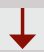

Score de I'ICSA

Abréviations : GAC, Bien manger avec le Guide alimentaire canadien; ICSA, Indice canadien de saine alimentation QFA-C II, Questionnaire canadien de fréquence alimentaire II; SAS, logiciel Statistical Analysis System.

Plusieurs sources de données ont été utilisées pour le calcul des nouvelles variables : la base de données sur les éléments nutritifs du QFA-C II, la base de données sur les éléments nutritifs du DHQ II des États-Unis la plus récente, le rapport de Santé Canada sur la classification des aliments dans le Fichier canadien sur les éléments nutritifs (FCEN) selon Bien manger avec le Guide alimentaire canadien (GAC) et sa base de données connexe (classification FCEN/ GAC) $)^{23,24}$, le fichier complet des aliments et des recettes provenant de l'Enquête sur la santé dans les collectivités canadiennes ${ }^{15}$ utilisé pour la mise au point de la base de données sur les éléments nutritifs du QFA-C II, la base de données sur les aliments et les éléments nutritifs du National Health and Nutrition Examination Survey utilisée pour mettre au point la base de données sur les éléments nutritifs du DHQ II et enfin la Food Patterns Equivalents Database $^{25}$.

Sur les 331 catégories d'aliments de la base de données sur les éléments nutritifs du QFA-C II, 302 ont été regroupées en quatre grandes familles en fonction de leur composition (ingrédients). Nous avons créé quatre algorithmes de complexité variable pour répartir les catégories d'aliments en fonction des composantes de l'ICSA (équivalents des groupes alimentaires) : 1) aliments simples, 2) aliments composés, 3) aliments avec ajout de matières grasses et 4) aliments d'exception (figure 2). Les 29 groupes alimentaires restants ont été répartis individuellement. La catégorie des aliments simples correspond aux aliments du QFA-C II constitués principalement d'aliments de base (lait, bananes, poivrons, pains, viande, etc.). La catégorie des aliments composés correspond aux aliments du QFA-C II constitués principalement d'un mélange de plusieurs aliments (pâtes alimentaires avec sauce à la viande, mélanges à base de poulet [sandwiches], pizza avec légumes et viande, etc.). La catégorie des aliments avec matières grasses ajoutées correspond aux aliments du QFA-C II composés principalement d'aliments simples auxquels on a ajouté des matières grasses pendant la cuisson ou la préparation (p. ex. poivrons avec matière grasse ajoutée). La catégorie des aliments d'exception est constituée des aliments du QFA-C II qui habituellement ne sont pas consommés seuls (sauces, tartinades, condiments, etc.).

Pour chacune des 302 catégories d'aliments, nous avons répertorié les aliments les plus souvent cités (trois au maximum) dans le fichier des aliments et des recettes de l'Enquête sur la santé dans les collectivités canadiennes (ci-dessous appelés aliments principaux) afin de représenter la catégorie d'aliments correspondante du QFA-C II servant à définir les équivalents des groupes alimentaires. Nous avons choisi les deux ou trois aliments principaux dans le cas où plusieurs aliments présentant des profils nutritionnels semblables avaient été déclarés à des fréquences de consommation similaires. Dans le cas d'aliments principaux multiples, nous avons d'abord estimé le poids moyen, en grammes, d'une portion du GAC puis nous avons pondéré la répartition par groupes alimentaires en conséquence.

Les aliments principaux sélectionnés dans le fichier des aliments et des recettes de l'Enquête sur la santé dans les collectivités canadiennes ont ensuite fait l'objet d'un examen plus approfondi pour s'assurer qu'ils étaient suffisamment représentatifs des catégories d'aliments correspondantes du QFA-C II, et ce, en comparant leurs profils nutritionnels à ceux des catégories de la base de données sur les éléments nutritifs du QFA-C II. La teneur en nutriments clés (énergie [kcal], sucre total [g], matières grasses totales [g], graisses saturées totales [g] et sodium [mg]) a été comparée pour une quantité équivalant à 100 grammes d'aliments. Les aliments principaux ont été considérés comme suffisamment représentatifs si la différence entre les valeurs nutritionnelles provenant du fichier de l'Enquête sur la santé dans les collectivités canadiennes et celles de la base de données sur les éléments nutritifs du QFA-C II était inférieure ou égale à $5 \%$. Dans les cas où la différence était supérieure à $5 \%$, les autres aliments les plus souvent déclarés dans la liste ont été pris en compte, jusqu’à obtention d'une représentativité suffisante (en incluant au maximum les trois premiers aliments déclarés par catégorie d'aliments du QFA-C II). Cette approche, assez conservatrice, a été considérée comme raisonnable par les auteurs ayant qualité d'experts (MM, IR, IM et IC).

Nous avons examiné en réalité un nombre variable de profils nutritionnels pour sélectionner un aliment principal lorsque les 
FIGURE 2

Algorithmes de base

Catégories d'aliments du QFA-C I

$\mathrm{N}=331$

Tous les groupes alimentaires du QFA-C II sont répartis en 4 groupes mutuellement exclusifs.

\begin{tabular}{|c|}
\hline $\begin{array}{c}\text { Aliments simples } \\
\mathrm{N}=208\end{array}$ \\
\hline \hline \\
Étape 1 : Déterminer les aliments \\
principaux dans le fichier de l'ESCC. \\
\hline \hline \\
Étape 2 : Rechercher les aliments \\
principaux dans la classification \\
FCEN/GAC pour déterminer le poids \\
en grammes (g) d'une portion \\
du GAC. \\
\hline Étape 3 : Comparer le \\
profil nutritionnel des aliments \\
recensés dans la classification FCEN/ \\
GAC au profil nutritionnel des \\
aliments de la base de données sur \\
les éléments nutritifs du QFA-C II. \\
\hline
\end{tabular}

Étape 4 : Déterminer la répartition des groupes alimentaires du GAC pour les aliments primaires dans la base de données de la classification FCEN/GAC.

Étape 5 : Diviser le poids (g) de chaque portion du QFA-C II par le poids (g) d'une (1) portion du GAC de l'aliment principal indiqué à l'étape 2.

Étape 6 : Dans la base de données sur les éléments nutritifs du QFA-C II, attribuer la valeur à la/aux variable(s) pertinente(s) de la portion équivalente du GAC indiquées à l'étape 4. Pour toutes les autres variables des portions équivalentes du GAC, attribuer la valeur « 0 ».

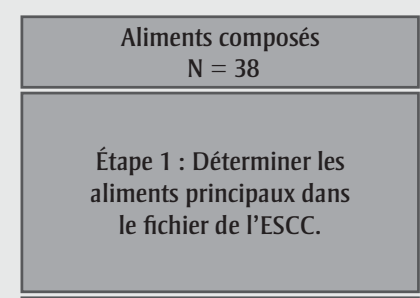

Étape 2 : Rechercher les aliments principaux dans le fichier complet de la MPED

et de la FPED du DHQ II et

déterminer au maximum

3 équivalents principaux

(mesurés en tasse américaine

ou en onces pour $100 \mathrm{~g}$ ).

Étape 3 : Convertir les équivalents MPED/FPED en poids gradué en grammes pour chaque portion du QFA-C II (g).

Étape 4 : Déterminer le poids (g) d'une (1) portion du GAC et d'une (1) portion américaine pour chacun des aliments primaires de la MPED et la FPED identifiés à l'étape 2 , dans l'outil de classification FCEN/GAC et dans le guide de l'utilisateur de la MPED et la FPED, respectivement.

Étape 5 : À l'aide des valeurs déterminées à l'étape 4 , ajuster le poids de la portion standard américaine afin qu'il reflète le poids standard d'une portion du GAC pour les valeurs de la MPED et la FPED déterminées à l'étape 3. Le résultat correspond au nombre de portions équivalentes du GAC.

Étape 6 : Dans la base de données sur les éléments nutritifs du QFA-C II, attribuer des portions équivalentes du GAC aux variables pertinentes correspondant aux répartitions de la MPED et la FPED. Pour toutes les autres variables des portions équivalentes du GAC, attribuer la valeur « $0 »$.
Aliments avec matière grasse ajoutée $\mathrm{N}=19$

Étape 1 : Déterminer les aliments principaux dans le fichier de l'ESCC à l'aide des groupes alimentaires du QFA-C II sans les aliments auxquels on a ajouté des matières grasses.

Étape 2 : Déterminer le ou les groupe(s) alimentaire(s) du GAC pour la répartition par niveaux des aliments principaux dans la classification FCEN/GAC

Étape 3 : Déterminer les grammes de matière grasse ajoutée en soustrayant le poids (g) du groupe des aliments sans matière grasse ajoutée du QFA-C II du groupe des aliments avec matière grasse ajoutée dans la base de données sur les éléments nutritifs du QFA-C II.

Étape 4 : Déterminer le type principal de matière grasse ajoutée (saturée ou non) à l'aide de la base de données sur les éléments nutritifs du QFA-C II.

Étape 5 : Pour les lipides insaturés utilisés comme matière grasse ajoutée, utiliser le poids en grammes du groupe alimentaire avec matière grasse ajoutée du QFA-C II et répéter les étapes 3 à 6 indiquées pour les « aliments simples ».

Étape 6 : Pour les lipides saturés utilisés comme matière grasse ajoutée, utiliser le poids en grammes du groupe alimentaire sans matière grasse ajoutée du QFA-C II et répéter les étapes 3 à 6 indiquées pour les « aliments simples». De plus, calculer la valeur calorique des lipides saturés ajoutés et affecter cette valeur à la variable « autres aliments » du GAC.

\begin{tabular}{c} 
Aliments d'exception \\
$\mathrm{N}=37$ \\
\hline
\end{tabular}

Étape 1 : Exécuter les étapes 1 à 4 comme pour les « aliments simples 》 pour les aliments classés 7000,8000 et 9999 dans la classification FCEN/ GAC.

Étape 2 : Les aliments codés 7000 et 9999 ne sont attribués à aucune des variables des équivalents de groupes alimentaires du GAC.

Étape 3 : Aliments codés 8000 : évaluer la teneur en sucre, en gras et en sodium du groupe du QFA-C II à l'aide des $\mathrm{QR}$, de la méthode et des maximums indiqués dans la classification du FCEN/GAC pour les niveaux.

Étape 4 : Si l'aliment fait partie du niveau 4, attribuer la valeur calorique à la variable « Autres aliments ». Pour toutes les autres variables, attribuer la valeur « 0 ». Si l'aliment fait partie des niveaux 1 à 3, répéter les étapes 2 à 6 indiquées pour les « aliments composés ».

Abréviations : DHQ II, Diet History Questionnaire II; ESCC, Enquête sur la santé dans les collectivités canadiennes; FCEN/GAC, Outil de classification du Fichier canadien sur les éléments nutritifs de Santé Canada selon le GAC; FPED, Food Patterns Equivalents Database; GAC, Bien manger avec le Guide alimentaire canadien; ICSA, Indice canadien de saine alimentation; MPED, MyPyramid Equivalents Database; QFA-C II, Questionnaire canadien de fréquence alimentaire II; QR, quantités de référence (tirées du FCEN/GAC).

Remarques : 29 groupes alimentaires n'ont suivi aucun des algorithmes, soit parce que les aliments ne se trouvaient pas dans le FCEN/GAC, soit parce que les aliments du groupe du QFA-C II ne pouvaient pas être déterminés, soit parce qu'il y avait conflit important dans la répartition des niveaux pour les aliments les plus fréquemment déclarés. Ces groupes alimentaires ont été analysés individuellement par au moins trois examinateurs.

Selon l'outil FCEN/GAC, 7000 = « autres aliments recommandés dans le GAC », 8000 = « recettes non classées » et 9999 = « aliments et boissons non classés ». 
aliments les plus fréquemment déclarés avaient été rapportés avec des fréquences similaires. Nous avons en ce cas examiné les principaux éléments nutritifs des aliments le plus souvent déclarés afin de déterminer les similitudes entre leurs profils nutritionnels. Si les profils nutritionnels différaient de $10 \%$ ou plus, jusqu'à cinq aliments ont pu être pris en compte pour l'analyse. Nous avons alors calculé des équivalents des groupes alimentaires pour chaque aliment puis établi la moyenne de leurs valeurs nutritionnelles afin de fixer une valeur finale. Nous avons rencontré d'autres cas exceptionnels dans l'algorithme des aliments composés, ce qui a conduit les coauteurs à examiner jusqu'à 20 profils nutritionnels afin de déterminer par consensus les aliments principaux les plus représentatifs. Cette manière de procéder visait à tenir compte de la diversité des aliments inclus dans les aliments composés du QFA-C II. Deux coauteures (IM et IR) ont examiné au hasard $10 \%$ des variables des équivalents de groupes alimentaires dans le cadre d'une démarche de vérification de la qualité. Pour ce faire, elles ont recalculé manuellement les équivalents des groupes alimentaires pour s'assurer que les algorithmes étaient correctement appliqués.

La classification FCEN/GAC a été établie par Santé Canada pour évaluer dans quelle mesure la population canadienne se conforme aux recommandations du GAC quant à la consommation d'aliments de chaque groupe alimentaire ${ }^{23,24}$. La classification du GAC a été appliquée au FCEN (2001b) ${ }^{26}$, qui est la base de données sur la composition nutritionnelle des aliments déclarés utilisée dans l'Enquête sur la santé dans les collectivités canadiennes. La classification FCEN/GAC attribue à chaque aliment du FCEN un groupe et un sous-groupe alimentaire du GAC. Les aliments du FCEN sont classés en " niveaux » selon leur conformité aux recommandations du GAC au sein de chacun des quatre grands groupes alimentaires du GAC (légumes et fruits, produits céréaliers, lait et substituts, viande et substituts) en tenant compte de leur teneur en gras, en sucre et en sodium ${ }^{23,24}$. Les niveaux 1 et 2 sont des " aliments conformes aux recommandations du GAC ", les aliments de niveau 3 sont des " aliments en partie conformes aux recommandations du GAC » et les aliments de niveau 4 sont les " aliments non conformes aux recommandations du GAC $»^{23,24}$.

Nous avons utilisé la classification FCEN/ GAC et la base de données connexe pour déterminer le poids d'une portion standard au sens du GAC d'un aliment principal et le groupe alimentaire du GAC auquel cet aliment appartient (c.-à-d. à quel équivalent du groupe alimentaire le faire correspondre). Tous les aliments de niveau 4 et ceux codés dans la classification FCEN/GAC comme " autres aliments ", "substituts de repas et suppléments » et " aliments et breuvages non classés » ont été considérés comme appartenant à l'équivalent de groupe alimentaire " autres aliments ». Lorsqu'un aliment principal était absent de la classification FCEN/GAC, ou si son type exact était inconnu (p. ex. "pain » sans autre description), nous avons utilisé le poids standard indiqué dans le $\mathrm{GAC}^{14}$ (le poids d'une portion de pain dans le GAC est par exemple de $30 \mathrm{~g}$ ) et les auteurs se sont entendus pour déterminer le groupe alimentaire du GAC le plus approprié dans le cas d'un aliment principal ne figurant ni dans la classification FCEN/GAC ni dans les bases de données du DHQII ou du National Health and Nutrition Examination Survey.

Pour les aliments principaux multiples et en cas de discordance de classification entre les niveaux et les aliments principaux, nous avons fait le choix du niveau correspondant le mieux aux aliments principaux (en se basant sur la classification de niveau la plus fréquente). Lorsque les algorithmes prédéfinis n’ont pas pu être utilisés en raison de données manquantes (aliments principaux ne figurant pas dans la base de données de la classification FCEN/ GAC, par exemple) ainsi que pour les aliments codés dans la classification FCEN/ GAC comme des "recettes ", nous avons utilisé des aliments ou des ingrédients similaires pour déterminer le niveau approprié de catégorie du QFA-C II. Dans ces cas-là, les auteurs ont décidé, après discussion, du classement dans le groupe approprié.

Pour l'algorithme des aliments composés, nous avons utilisé la classification des groupes alimentaires des bases de données américaines Food Patterns Equivalents Database (FPED) et MyPyramid Equivalents Database (MPED) dans la base de données des aliments et des recettes du National Health and Nutrition Examination Survey afin de sélectionner les équivalents de groupes alimentaires appropriés. Ce sont les groupes alimentaires de la MPED et non ceux de la FPED qui ont été utilisés pour les produits céréaliers car les méthodes employées pour déterminer leurs valeurs correspondaient davantage à notre méthode d'établissement des équivalents de groupes alimentaires $^{26}$.

Pour déterminer les ingrédients des aliments composés (types de viande, de légumes et de céréales, etc.), nous avons utilisé les descripteurs des aliments principaux contenus dans le fichier des aliments et des recettes de l'Enquête sur la santé dans les collectivités canadiennes. Dans certains cas, le choix a reposé sur les connaissances des auteurs quant aux ingrédients habituellement inclus dans les recettes d'aliments composés (p. ex., pour les rouleaux de printemps, on a présumé que le chou était le principal contributeur au groupe alimentaire des légumes et des fruits). Pour vérifier la validité de ces hypothèses, nous avons comparé les profils nutritionnels des aliments principaux dans la base de données du National Health and Nutrition Examination Survey aux profils nutritionnels de cette catégorie d'aliments dans la base de données sur les éléments nutritifs du QFA-C II en portant une attention particulière aux principaux éléments nutritifs mentionnés précédemment. Une différence approximative d'au plus $20 \%$ entre les deux profils nutritionnels a été jugée acceptable étant donné la différence de composition des aliments entre les États-Unis et le Canada ${ }^{17}$. Pour chaque aliment principal, les trois valeurs les plus élevées de la FPED (et dans le cas des céréales, de la MPED) ont été ajustées pour tenir compte du poids total en grammes de chaque ingrédient dans chacune des six portions du QFA-C II. Nous avons ensuite ajusté les poids en grammes lors de la conversion des poids des portions standards américaines ${ }^{26}$ en poids des portions du $\mathrm{GAC}^{24}$.

Il est resté 29 catégories d'aliments du QFA-C II qui n’ont pas pu être réparties dans les quatre groupes alimentaires mutuellement exclusifs pour l'une des raisons suivantes : ces aliments ne se trouvaient pas dans la classification FCEN/GAC, nous n'avons pas pu définir les aliments inclus dans la catégorie du QFA-C II, il y a eu discordance importante de classement par niveau pour les aliments les plus fréquemment déclarés de cette catégorie, ou encore ces aliments n'étaient pas pertinent pour calculer l'ICSA (p. ex. épices, café, thé). Chacune de ces 29 catégories d'aliments a été analysée séparément.

Comme aucun nom de marque n'était cité dans le fichier des aliments et des recettes 
de l'Enquête sur la santé dans les collectivités canadiennes pour les céréales de petit déjeuner prêtes à manger, nous n'avons pas pu à partir de la classification FCEN/ GAC savoir si elles relevaient de la catégorie des grains entiers. Nous avons donc conçu un algorithme pour limiter la sousestimation des grains entiers. Nous avons calculé un poids moyen pour toutes les céréales de type " prêtes à manger » dans la base de données de la classification FCEN/ GAC afin de déterminer le poids standard en grammes de céréales de ce type dans les catégories d'aliments du QFA-C II. Nous nous sommes ensuite servis des calculs de répartition des grains entiers pour les céréales «prêtes à manger » des bases de données du DHQ II pour calculer la quantité de grains entiers. Nous avons employé les groupes de la MPED « ensemble des céréales » et " grains entiers » dans la formule

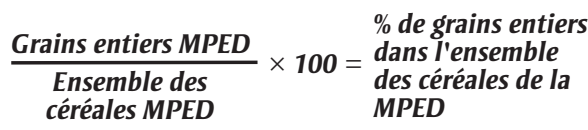

puis nous avons multiplié le pourcentage de grains entiers dans l'ensemble des céréales de la MPED par la valeur de l'ensemble des céréales au Canada (à l'aide de l'algorithme des aliments simples) pour déterminer la valeur de la variable de l'équivalent du groupe alimentaire des grains entiers.

Nous avons ajouté à la base de données sur les éléments nutritifs du QFA-C II les équivalents des groupes alimentaires pour toutes les catégories d'aliments du QFA-C II. Nous avons estimé l'apport quotidien des équivalents de groupes alimentaires pour chaque groupe alimentaire grâce au logiciel DietCalc (version 1.5.1), le programme d'analyse des éléments nutritifs du QFA-C II. Nous avons utilisé le logiciel SAS pour analyser les résultats du QFA-C II et calculer l'ICSA (le code SAS ayant servi à déterminer l'ICSA est disponible auprès des auteurs).

\section{Échantillon et procédure d'essai}

Des participants recrutés dans le cadre de l'étude Pathways to Health ${ }^{21}$ - dont l'objectif est d'examiner la relation entre la qualité du régime alimentaire et l'environnement du quartier chez les adultes - ont répondu au QFA-C II. L'étude, qui a commencé en avril 2014, s'appuie sur un échantillon aléatoire stratifié à deux degrés d'adultes provenant de 12 quartiers de
Calgary (Alberta, Canada). Nous avons inclus dans cette analyse les 446 participants qui ont rempli le questionnaire QFA-C II en ligne en réponse à notre courrier d'invitation.

Toutes les analyses ont été stratifiées selon le sexe. Des statistiques descriptives (moyennes, écarts-types, médiane, minimum et maximum) ont été calculées pour les éléments suivants : score total de l'ICSA, score des composantes de l'ICSA, apport énergétique et nombre de portions du GAC. Nous avons analysé l'apport moyen en énergie et les portions du GAC afin de déterminer la vraisemblance des résultats en fonction d'une « connaissance a priori » des apports alimentaires pour des populations comparables.

Des rapports précédents montrent que les scores de l'ICSA varient en fonction des caractéristiques sociodémographiques (sexe, âge, origine ethnique, statut vis-à-vis de l'immigration, revenu du ménage, niveau de scolarité et tabagisme) ${ }^{8}$. Nous avons utilisé une analyse de la variance avec correction de Bonferonni post-hoc pour estimer les variations dans le score total de l'ICSA en fonction du groupe d'âge, de l'état matrimonial, du niveau de scolarité, du revenu brut du ménage, du tabagisme et de l'origine ethnique, et ce, pour l'ensemble de la population et selon le sexe. De plus, nous avons évalué la tendance linéaire des scores de l'ICSA pour les catégories de revenu du ménage et de niveau de scolarité, là aussi pour l'ensemble de la population et selon le sexe. Nous avons utilisé des tests t indépendants pour estimer les variations dans les scores des composantes de l'ICSA selon le sexe. Le seuil de signification statistique a été fixé à $\alpha<0,05$. Tous les tests de signification ont été effectués au moyen du logiciel statistique Stata, version 13.0 (StataCorp LP, College Station, Texas, É.-U.).

\section{Résultats}

Notre échantillon était composé de 172 hommes et 274 femmes. La majorité des participants étaient mariés ou vivaient avec un partenaire, avaient au moins un diplôme d'études secondaires, vivaient dans un ménage dont le revenu était supérieur à 60000 \$, ne fumaient pas et étaient d'origine ethnique blanche (tableau 2).
La répartition des scores de l'ICSA s'est révélé proche de la normale (données non présentées). Le score quotidien moyen de l'ICSA était de 64,4 (écart-type : 10,8) (tableau 2). Les hommes $(61,5$ [10,5]) affichaient des scores inférieurs à ceux des femmes $(66,3[10,6])$ et les fumeurs $(56,9$ $[11,8])$ des scores inférieurs à ceux des non-fumeurs $(64,7[10,6])$. Nous avons observé en outre une tendance linéaire croissante statistiquement significative en fonction du niveau de scolarité. Le fait de ne pas fumer et un niveau d'études supérieur étaient également associés à des scores d'ICSA plus élevés chez les femmes, mais seul le fait de ne pas fumer était associé à un score plus élevé chez les hommes.

La moyenne (écart-type) de l'apport énergétique quotidien était de 1650 (717) kcal (tableau 3). Le nombre moyen le plus élevé de portions quotidiennes moyennes du GAC concernait la consommation totale de légumes et de fruits $6,3(3,2)$, tandis que le plus faible concernait les grains entiers $0,4(0,4)$. Le nombre moyen de portions quotidiennes s'est révélé inférieur aux recommandations du GAC en fonction de l'âge et du sexe pour tous les groupes alimentaires du GAC.

Les scores des composantes de l'ICSA sont présentés dans le tableau 3. Comparativement aux hommes, les femmes ont obtenu des scores significativement plus élevés $(p<0,05)$ pour les composantes « lipides insaturés ", " sodium ", " viande et substituts » et « autres aliments », tandis que les hommes ont obtenu un score significativement plus élevé $(p<0,05)$ pour la composante « lipides saturés ».

La figure 3 illustre l'aspect multidimensionnel de l'ICSA et les différences de conformité aux recommandations concernant la suffisance des apports des groupes alimentaires et la modération dans les apports non recommandés. En moyenne, aucune des recommandations pour ces composantes n'est respectée à $100 \%$, les hommes et les femmes se conformant de manière similaire aux apports recommandés pour les composantes de l'ICSA. Les grains entiers sont la composante ayant obtenu le score le plus faible, tandis que les composantes dont les scores sont les plus élevés sont les lipides insaturés, les fruits entiers, les légumes vert foncé et orangés et la consommation totale de fruits et légumes. 
TABLEAU 2

Caractéristiques individuelles des participants et répartition des scores quotidiens totaux moyens de l'ICSA, selon le sexe

\begin{tabular}{|c|c|c|c|c|c|c|c|c|c|c|c|c|}
\hline & \multicolumn{4}{|c|}{ Total } & \multicolumn{5}{|c|}{ Hommes } & \multicolumn{3}{|c|}{ Femmes } \\
\hline & $\%$ & $\begin{array}{l}\text { Moyenne } \\
\text { (ET) }\end{array}$ & Médiane & Min, max & n & $\begin{array}{l}\text { Moyenne } \\
\text { (ET) }\end{array}$ & Médiane & Min, max & n & $\begin{array}{l}\text { Moyenne } \\
\text { (ET) }\end{array}$ & Médiane & Min, max \\
\hline \multicolumn{13}{|l|}{ Âge (ans) } \\
\hline 21 à 39 & 20,4 & $65,6(10,1)$ & 66,4 & $37,1,88,5$ & 23 & $60,0(10,5)$ & 62,9 & $37,1,77,4$ & 68 & $67,4(9,4)^{b}$ & 68,4 & $46,1,88,5$ \\
\hline 60 et plus & 34,8 & $64,1(11,0)$ & 65,1 & $31,4,84,2$ & 81 & $61,9(11,0)$ & 63,0 & $31,4,79,2$ & 74 & $66,5(10,7)$ & 67,6 & $7,1,84,2$ \\
\hline \multicolumn{13}{|l|}{ État matrimonial } \\
\hline $\begin{array}{l}\text { Marié ou vivant avec } \\
\text { un(e) conjoint(e) }\end{array}$ & 78,5 & $65,1(10,6)$ & 65,7 & $31,4,88,5$ & 141 & $61,8(10,3)$ & 62,1 & $31,4,79,2$ & 209 & $67,3(10,3)^{b}$ & 68,5 & $36,9,88,5$ \\
\hline $\begin{array}{l}\text { Toutes les autres } \\
\text { situations }\end{array}$ & 21,5 & $62,1(11,0)$ & 64,2 & $30,2,84,0$ & 31 & $60,1(11,8)$ & 63,9 & $31,5,75,8$ & 65 & $62,1(11,0)$ & 64,2 & $30,1,84,0$ \\
\hline $\begin{array}{l}\text { Diplôme d'études } \\
\text { collégiales/ } \\
\text { professionnelles/ } \\
\text { école de métier }\end{array}$ & 17,5 & $64,2(11,8)$ & 65,5 & $31,4,84,3$ & 31 & $61,7(11,6)$ & 63,2 & $31,4,78,0$ & 47 & $65,9(11,7)$ & 67,0 & $37,0,84,3$ \\
\hline Université & 70,0 & $65,2(10,6)^{c}$ & 66,3 & $30,2,88,5$ & 120 & $61,8(10,5)$ & 63,3 & $31,5,79,2$ & 192 & $67,2(10,1)^{b, c}$ & 68,5 & $30,2,88,5$ \\
\hline \multicolumn{13}{|c|}{ Revenu brut du ménage } \\
\hline 0 à $59999 \$$ & 8,7 & $63,1(10,4)$ & 63,0 & $43,3,84,2$ & 18 & $64,7(10,6)$ & 64,7 & $43,3,79,2$ & 21 & $61,7(10,3)$ & 59,6 & $45,0,59,6$ \\
\hline 60000 \$ à $119999 \$$ & 33,9 & $63,5(10,7)$ & 65,5 & $31,4,85,1$ & 61 & $59,0(10,6)$ & 60,1 & $31,4,79,2$ & 90 & $66,6(9,6)^{b}$ & 68,2 & $31,4,79,2$ \\
\hline $120000 \$$ et plus & 43,3 & $65,7(10,7)$ & 67,0 & $30,2,88,5$ & 76 & $62,6(10,4)$ & 63,5 & $31,4,78,9$ & 117 & $67,7(10,5)^{b}$ & 68,9 & $30,2,88,5$ \\
\hline Autre & 6,5 & $65,4(10,0)$ & 66,1 & $40,0,79,3$ & 57 & $63,8(7,8)$ & 64,2 & $49,6,76,6$ & 260 & $67,1(12,0)$ & 69,7 & $40,0,73,3$ \\
\hline
\end{tabular}

Abréviations : ET, écart-type; ICSA, Indice canadien de saine alimentation.

${ }^{a}$ Différence significative selon le sexe, $p<0,05$, test $t$ indépendant.

${ }^{\mathrm{b}}$ Différence significative selon le sexe au sein des groupes sociodémographiques, $p<0,05$, analyse de la variance avec correction de Bonferonni post-hoc.

' Tendance linéaire significative, $p<0,05$, entre les groupes sociodémographiques, analyse de la variance avec test post-hoc.

${ }^{d}$ Différence significative entre les groupes sociodémographiques, $p<0,05$, analyse de la variance avec correction de Bonferonni post-hoc.

\section{Analyse}

Nous avons décrit les méthodes de création d'équivalents des groupes alimentaires (variables des portions du GAC) pour l'estimation de l'ICSA à l'aide de la base de données sur les éléments nutritifs du QFA-C II. Nous avons élaboré des algorithmes pour créer les variables des équivalents des groupes alimentaires et nous les avons appliqués aux aliments les plus consommés du fichier des aliments et des recettes de l'Enquête sur la santé dans les collectivités canadiennes en lien avec les questions du QFA-C II. Cette approche a constitué une stratégie solide et efficace, car les profils nutritionnels obtenus à partir des catégories alimentaires du QFA-C II correspondaient aux aliments qui contribuent majoritairement au régime alimentaire des Canadiens ${ }^{15}$ et se sont donc révélés représentatifs de ces catégories. La moyenne des scores de l'ICSA chez les participants à l'étude Pathways to Health était comparable, quoique légèrement supérieure, aux valeurs calculées précédemment pour la population canadienne ${ }^{8}$. Conformément aux données canadiennes antérieures ${ }^{8}$, nous avons constaté des différences dans les scores de l'ICSA en fonction du sexe, du tabagisme et du niveau de scolarité.
La fourchette et la distribution normale observées pour les apports quotidiens moyens par équivalent des groupes alimentaires donnent à penser que la méthode appliquée permet une estimation raisonnable de ces apports, étant donné les similitudes avec les rapports déjà publiés pour la population canadienne ${ }^{8}$. Les apports en fruits et légumes, en fruits entiers, en légumes vert foncé et orangés et en portions de viande se sont trouvés légèrement décalés vers la gauche, ce qui était prévisible étant donné que les régimes nordaméricains sont généralement riches en protéines animales ${ }^{27,28}$ et que la désirabilité sociale entraine souvent une surestimation 
TABLEAU 3

Répartition de l'apport quotidien déclaré des équivalents de groupes alimentaires (en portions du Guide alimentaire canadien), scores des composantes de l'Indice canadien de saine alimentation et apport énergétique, selon le sexe

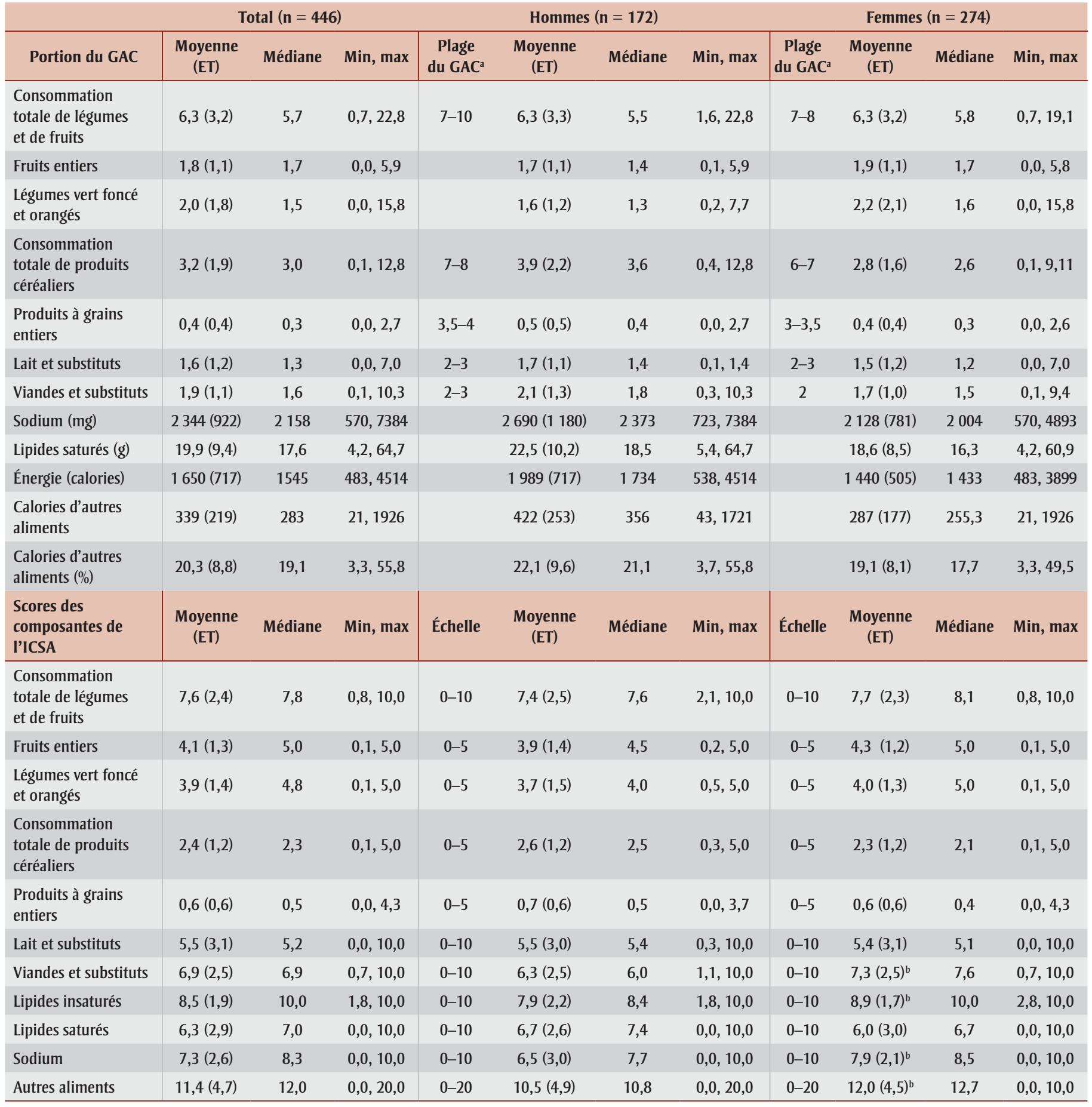

Abréviations : ET, écart-type; GAC, Bien manger avec le Guide alimentaire canadien; ICSA, Indice canadien de saine alimentation.

Remarque : Plage du GAC = nombre recommandé de portions du Guide alimentaire par jour, adultes de 19 ans et plus.

${ }^{a}$ Recommandation du GAC pour les adultes de 19 ans et plus.

${ }^{\mathrm{b}}$ Différence significative selon le sexe, $p<0,05$. 
FIGURE 3

Graphique en étoile montrant les scores quotidiens moyens des composantes de l'ICSA pour l'ensemble de l'échantillon et selon le sexe, par rapport à un score ICSA parfait

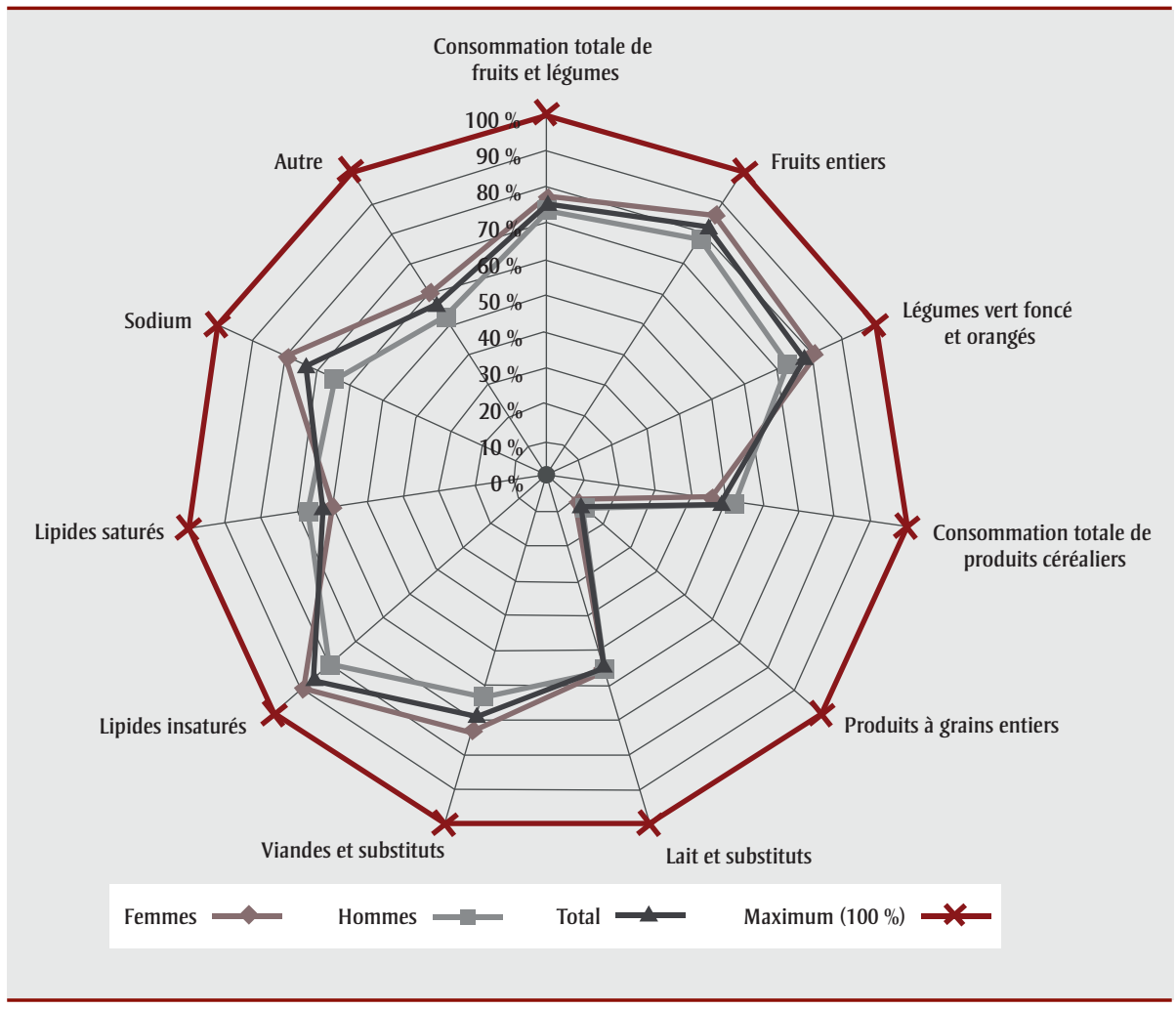

de la consommation de fruits et légumes ${ }^{29}$. Comme dans les études antérieures sur les habitudes alimentaires canadiennes, les femmes ont déclaré un nombre quotidien moyen plus élevé de portions de fruits et légumes que les hommes ${ }^{30,31}$, tandis que les hommes ont déclaré un nombre quotidien moyen plus élevé de portions de viande et substituts $^{30}$. L'apport de céréales et de grains entiers, mesuré par l'apport quotidien moyen de l'équivalent GAC, s'est révélé faible. La faible consommation de céréales et de grains entiers pourrait s'expliquer par la prévalence accrue de la sensibilité au gluten non cœliaque ${ }^{32}$ et la popularité croissante des régimes alimentaires sans gluten et à faible teneur en glucides ${ }^{33-35}$, susceptibles de limiter la consommation de produits céréaliers. Notons enfin que la répartition des apports par équivalent de groupes alimentaires est semblable à celle observée antérieurement en Alberta ${ }^{20}$.

Le score quotidien moyen de l'ICSA $(64,4)$ s'est révélé un peu plus élevé que celui observé précédemment à l'échelle nationale $(58,8)^{8}$ et en Alberta (hommes : 51; femmes : 56) ${ }^{20}$. La différence entre les scores calculés dans notre étude et ceux d'autres études reflète sans doute une différence dans les modèles d'échantillonnage et les méthodes de collecte de données. Quoi qu'il en soit, dans notre étude, la différence dans les scores de l'ICSA en fonction des diverses caractéristiques sociodémographiques concorde avec les résultats d'une autre étude canadienne ayant fait état de différences selon le sexe, le tabagisme et le niveau de scolarité ${ }^{8}$. En ce qui concerne les scores associés aux composantes de l'ICSA, à l'exception de la consommation totale de légumes et fruits, de viande et substituts et de grains entiers, toutes les valeurs minimales et maximales possibles ont été observées, ce qui prouve que notre méthode fondée sur les aliments principaux permet d'obtenir un éventail complet de scores.

\section{Limites}

Nous savons que la plupart des méthodes d'évaluation de l'alimentation comportent une part de sous-déclaration ${ }^{36,37}$ et que celle-ci a probablement joué un rôle dans notre étude ${ }^{38}$. Il a été constaté que les questionnaires de fréquence alimentaire étaient associés à une sous-déclaration importante des apports énergétiques en comparaison des mesures objectives obtenues avec la méthode de l'eau doublement marquée ${ }^{38-40}$. On s'attend donc à ce que ce phénomène de sous-déclaration soit aussi présent avec le QFA-C II, la sous-déclaration dans le cadre du QFA-C II ayant probablement entraîné une sous-estimation de l'apport énergétique global. Certaines données indiquent que les déclarations erronées varient selon le type d'aliments dans les questionnaires de fréquence alimentaire ${ }^{41,42}$. Par exemple, les légumes et les fruits ont tendance à être surestimés, tandis que les sucres, les bonbons, les confitures et certains produits céréaliers sont sousestimés ${ }^{42}$. De plus, il est possible que la désirabilité sociale ait engendré un biais dans les réponses, ceux qui ont choisi de répondre au questionnaire pouvant avoir sous-déclaré certains groupes d'aliments considérés " malsains » par les médias populaires (les produits céréaliers, par exemple). Les déclarations erronées ont donc pu entraîner aussi bien une sous-estimation qu'une surestimation des composantes " suffisance » et " modération » et du score total de l'ICSA. Même si dans notre étude l'apport quotidien moyen des équivalents des groupes alimentaires et les estimations de l'ICSA sont dans l'ensemble conformes aux portions actuelles du $\mathrm{GAC}^{30}$ et aux répartitions des scores de ''ICSA $^{8}$, certaines estimations sont faibles (surtout en ce qui concerne les céréales et les grains entiers). Il pourrait s'agir d'une conséquence de l'impossibilité d'identifier les aliments principaux due à l'absence de noms de marques et à la difficulté à estimer le contenu en céréales des aliments composés, ou encore au fait qu'on a utilisé uniquement les aliments les plus consommés rapportés dans l'Enquête sur la santé dans les collectivités canadiennes et associés aux questions du QFA-C II.

Les valeurs seuil pour garantir la représentativité suffisante des aliments principaux (différence de $5 \%$ ou moins) et l'écart dans la composition des aliments composés tirés de la MPED et de la FPED (environ $20 \%$ ) ont été fixées par les auteurs. Le choix de limites peut-être trop larges ou trop conservatrices ont pu entraîner une mauvaise répartition d'un groupe alimentaire du QFA-C II dans les variables de l'ICSA.

\section{Conclusion}

L'ajout d'équivalents des groupes alimentaires à la base de données sur les éléments nutritifs du QFA-C II permet aux chercheurs 
de comparer les apports quotidiens moyens des différents groupes alimentaires aux recommandations du GAC pour une saine alimentation. De plus, le calcul de l'ICSA permet aux chercheurs d'examiner la relation entre la qualité du régime alimentaire et le risque de maladie chronique au moyen d'un questionnaire conçu spécifiquement pour la population canadienne.

\section{Remerciements}

Cette recherche a été financée par des fonds du Conseil de recherches en sciences humaines (CRSH), des Instituts de recherche en santé du Canada (IRSC), de la Fondation canadienne pour l'innovation (FCI) et de Statistique Canada versés au Réseau canadien des Centres de données de recherche (RCCDR).

Les allocations de MM proviennent de la subvention de formation des IRSC pour les interventions auprès des populations pour la prévention des maladies chroniques : programme pancanadien (subvention 53893), du programme de bourses d'études supérieures du Canada au niveau de la maîtrise (IRSC) et d'une bourse William $\mathrm{H}$ Davies Medical Research Scholarship. GRM a reçu une bourse de nouveau chercheur des IRSC. AK a été soutenue par le programme des chercheurs-boursiers du Fonds de recherche du Québec-Santé (FRQS) et le programme des nouveaux chercheurs des IRSC. VH a reçu une bourse postdoctorale des IRSC et de Cancer pulmonaire Canada et est actuellement appuyée par la Société de recherche sur le cancer, le FRQS et le ministère de l'Économie, de la Science et de l’Innovation du Québec (MESI).

\section{Conflits d'intérêts}

Les auteurs déclarent n'avoir aucun conflit d'intérêts à déclarer.

\section{Contributions des auteurs et avis}

MM et IC ont conçu les méthodologies et les algorithmes utilisés pour créer les variables du Guide alimentaire canadien dans la base de données sur les éléments nutritifs du Questionnaire canadien de fréquence alimentaire II. MM a supervisé la création des variables du Guide alimentaire canadien. IM, IR, AK et VH ont vérifié les algorithmes et les méthodologies. VH a modifié le code SAS pour déterminer l'Indice canadien de saine alimentation à partir des données du QFA-C II. IM et IR ont contrôlé la qualité des nouvelles variables. GRM a formulé des conseils pour l'analyse. MM a supervisé la rédaction et la révision du manuscrit. Tous les auteurs ont contribué à l'interprétation des résultats et à la rédaction du manuscrit. Tous les auteurs ont lu et approuvé le manuscrit final.

Le contenu de cet article et les opinions qui y sont exprimées n'engagent que les auteurs et ne représentent pas forcément les opinions du Gouvernement du Canada. Bien que les travaux de recherche et les analyses soient fondés sur des données fournies par Statistique Canada, les avis exprimés ici ne sont pas représentatifs du point de vue de ce ministère.

\section{Références}

1. Alkerwi A. Diet quality concept. Nutrition. 2014;30(6):613-618. doi: 10.1016/j.nut.2013.10.001.

2. Anic GM, Park Y, Subar AF, Schap TE, Reedy J. Index-based dietary patterns and risk of lung cancer in the NIHAARP diet and health study. Eur J Clin Nutr. 2016;70(1):123-129. doi: 10.1038 /ejcn.2015.122.

3. Reedy J, Mitrou PN, Krebs-Smith SM, Wirfält E, Flood A, Kipnis V, et al. Index-based dietary patterns and risk of colorectal cancer in the NIH-AARP diet and health study. Am J Epidemiol. 2008;168(1):38-48. doi: 10.1093/aje /kwn097.

4. Bosire C, Stampfer MJ, Subar AF, Park Y, Kirkpatrick SI, Chiuve SE, et al. Index-based dietary patterns and the risk of prostate cancer in the $\mathrm{NIH}$ AARP diet and health study. Am J Epidemiol. 2013;177(6):504-513. doi: 10.3945/an.115.009746.

5. Chiuve SE, Fung TT, Rimm EB, Hu FB, McCullough $\mathrm{M}$, Wang $\mathrm{M}$, et al. Alternative dietary indices both strongly predict risk of chronic disease. J Nutr. Am Soc Nutrition. 2012; 142(6):1009-1018. doi: 10.3945/jn.111 .157222 .

6. Liese AD, Krebs-Smith SM, Subar AF, George SM, Harmon BE, Neuhouser ML, et al. The Dietary Patterns Methods Project: synthesis of findings across cohorts and relevance to dietary guidance. J Nutr. 2015;145(3):393-402.
7. Krebs-Smith SM, Subar AF, Reedy J. Examining dietary patterns in relation to chronic disease: matching measures and methods to questions of interest. Circulation. 2015;132:790-793. doi: 10.1161/CIRCULATIONAHA.115.018010.

8. Garriguet, D. La qualité de l'alimentation au Canada. Rapports sur la santé. 2009;20(3):41-52.

9. Kennedy ET, Ohls J, Carlson S, Fleming K. The healthy eating index: design and applications. J Am Diet Assoc. 1995;95(10):1103-1108. doi: 10.1016/S0002-8223(95)00300-2.

10. Dubois L, Girard M, Bergeron N. The choice of a diet quality indicator to evaluate the nutritional health of populations. Public Health Nutr. 2000; 3(3):357-365.

11. Glanville NT, McIntyre L. Diet quality of Atlantic families headed by single mothers. Can J Diet Pract Res. 2006; 67(1):28-35. doi: 10.3148/67.1.2006.28.

12. Shatenstein B, Nadon S, Godin C, Ferland G. Diet quality of Montrealarea adults needs improvement: estimates from a self-administered food frequency questionnaire furnishing a dietary indicator score. J Am Diet Assoc. 2005;105(8):1251-1260.

13. Woodruff SJ, Hanning RM, McGoldrick K, Brown KS. Healthy Eating Index-C is positively associated with family dinner frequency among students in grades 6-8 from Southern Ontario, Canada. Eur J Clin Nutr. 2010;64(5): 454-460. doi: 10.1038/ejcn.2010.14.

14. Santé Canada. Bien manger avec le Guide alimentaire canadien [Internet]. Ottawa (Ont.) : Santé Canada; 2007 [consultation le 23 octobre 2013]. En ligne à : http://www.hc-sc.gc.ca/fn-an /food-guide-aliment/order-commander /index-fra.php

15. Santé Canada. Enquête sur la santé dans les collectivités canadiennes, cycle 2.2, Nutrition (2004) - Guide d'accès et d'interprétation des données. Ottawa (Ont.) : Santé Canada; 2006.

16. Canadian DHQ II. Questionnaire canadien de fréquence alimentaire II (QFA-C II). Formulaires et fichiers Diet Calc [Internet]. 2015. En ligne à : https:// www.canadiandhqii.com/survey_ resources.html 
17. Csizmadi I, Boucher B, Lo Siou G, Massarrelli I, Rondeau I, Koushik A, et al. Using national dietary intake data to evaluate and adapt the US Diet History Questionnaire: the stepwise tailoring of an FFQ for Canadian populations. Public Health Nutr. 2016; 19(18):3247-3255. doi: 10.1017/S1368 980016001506 .

18. Lo Siou G, Csizmadi I, Boucher BA, et al. The comparative reliability and feasibility of the past-year Canadian Diet History Questionnaire II: comparison of the paper and web versions. Nutrients. 2017;9(2):E133. doi: 10.3390 /nu9020133.

19. Awadalla P, Boileau C, Payette Y, Idaghdour Y, Goulet J-P, Knoppers B, et al. Cohort profile of the CARTaGENE study: Quebec's population-based biobank for public health and personalized genomics. Int J Epidemiol. 2013; 42(5):1285-1299. doi: 10.1093/ije /dys 160.

20. Solbak NM, Xu JY, Vena JE, Csizmadi I, Whelan HK, Robson PJ. Diet quality is associated with reduced incidence of cancer and self-reported chronic disease: observations from Alberta's Tomorrow Project. Prev Med. 2017; 101:178-187. doi: 10.1016/j.ypmed.2017 .06 .009 .

21. McInerney M, Csizmadi I, Friedenreich CM, Nettle-Aguirre A, Sandalack BA, Potestio M, et al. Associations between neighbourhood food environment, neighbourhood socioeconomic status, and diet quality. BMC Public Health. 2016;16(1):984-999. doi: 10.1186/s12889 $-016-3631-7$.

22. McInerney, M. Associations between the neighbourhood food environment, neighbourhood socioeconomic status and diet quality in Canadian adults. Calgary (AB) : University of Calgary; 2016. En ligne à : http://hdl.handle .net/11023/2848.

23. Elvidge Munene L-A, Dumais L, Esslinger K, Jones-Mclean E, Mansfield E, Verreault M-F, et al. Un outil de surveillance pour évaluer les régimes alimentaires selon Bien manger avec le Guide alimentaire canadien. Rapports sur la santé. 2015; 26(11):13-22. doi: 10.1186/s12889-016-3631-7.
24. Santé Canada. L'élaboration et l'utilisation d'un outil de surveillance : La classification des aliments dans le Fichier canadien sur les éléments nutritifs selon Bien manger avec le Guide alimentaire canadien. Ottawa (Ont.) : Santé Canada; 2014. En ligne à : http://publications.gc.ca/collections /collection_2014/sc-hc/H164-158-2 -2014-fra.pdf

25. Bowman S, Clemens J, Friday J, Thoerig R, Moshfegh A. Food Patterns Equivalents Database 2011-12: Methodology and User Guide. Beltsville. 2014. En ligne à : https://www.ars.usda.gov /ARSUserFiles/80400530/pdf/fped /FPED_1112.pdf

26. Santé Canada. Le Fichier canadien sur les éléments nutritifs (FCÉN) 2001b [Internet]. Ottawa (Ont.) : Santé Canada; 2001. En ligne à : https://www .canada.ca/fr/sante-canada/services /aliments-nutrition/saine-alimentation /donnees-nutritionnelles / fichier -canadien-elements-nutritifs-fcen-2015 .html

27. Statistique Canada. Nutrition : Résultats de l'Enquête sur la santé dans les collectivités canadiennes (82-620-M) [Internet]. Ottawa (Ont.) : Statistique Canada; 2007. En ligne à : http://www .statcan.gc.ca/pub/82-620-m/2006002 /4053669-fra.htm

28. Daniel CR, Cross AJ, Koebnick C, Sinha R. Trends in meat consumption in the United States. Public Health Nutr. 2011;14(4):575-583. doi: 10.1017 /S1368980010002077.

29. Miller TM, Abdel-Maksoud MF, Crane LA, Marcus AC, Byers TE. Effects of social approval bias on self-reported fruit and vegetable consumption: a randomized controlled trial. Nutr J. 2008;7(18). doi: 10.1186/1475-2891 $-7-18$.

30. Garriguet, D. Les habitudes alimentaires des Canadiens. Rapports sur la santé. 2007;18(2):17-33.

31. Pérez CE. Consommation de fruits et de légumes. Rapports sur la santé. 2002;13(3):25-34.
32. DiGiacomo D V, Tennyson CA, Green $\mathrm{PH}$, Demmer RT. Prevalence of glutenfree diet adherence among individuals without celiac disease in the USA: results from the Continuous National Health and Nutrition Examination Survey 2009-2010. Scand J Gastroenterol. 2013;48(8):921-925. doi: 10.3109 /00365521.2013.809598.

33. Volland A. Gluten-free diet: a cure for some, a fad for most. U.S. news and world report. 2008;145(12):66-68.

34. Cadenhead K, Sweeny M. Gluten elimination diets: facts for patients on this food fad. BCMJ. 2013;55:161.

35. Moore LR. "But we're not hypochondriacs": The changing shape of glutenfree dieting and the contested illness experience. Soc Sci Med. 2014;105:7683. doi: 10.1016/j.socscimed.2014.01 .009 .

36. Grandjean AC. Dietary intake data collection: challenges and limitations. Nutr Rev. 2012;70 Suppl 2:S101-104. doi: 10.1111/j.1753-4887.2012.00545.x.

37. Shim J-S, Oh K, Kim HC. Dietary assessment methods in epidemiologic studies. Epidemiol Health. 2014;36: e2014009. doi: 10.4178/epih/e2014009.

38. Subar AF, Kipnis V, Troiano RP, Midthune D, Schoeller DA, Bingham $\mathrm{S}$, et al. Using intake biomarkers to evaluate the extent of dietary misreporting in a large sample of adults: the OPEN study. Am J Epidemiol. 2003; 158(1):1-13.

39. Prentice RL, Mossavar-Rahmani Y, Huang Y, Van Horn L, Beresford SAA, Caan B, et al. Evaluation and comparison of food records, recalls, and frequencies for energy and protein assessment by using recovery biomarkers. Am J Epidemiol. 2011;174(5): 597-603.

40. Freedman LS, Commins JM, Moler JE, Arab L, Baer DJ, Kipnis V, et al. Pooled results from 5 validation studies of dietary self-report instruments using recovery biomarkers for energy and protein intake. Am J Epidemiol. 2014;180(2)172-188. doi: 10.1093/aje /kwu116. 
41. Streppel MT, de Vries JHM, Meijboom $S$, Beekman $M$, de Craen AJM, Slagboom PE, et al. Relative validity of the food frequency questionnaire used to assess dietary intake in the Leiden Longevity Study. Nutr J. 2013;12(1):18. doi: 10.1186/1475-2891-12-75.

42. Marks GC, Hughes MC, van der Pols JC. Relative validity of food intake estimates using a food frequency questionnaire is associated with sex, age, and other personal characteristics. J Nutr. 2006;136(2):459-465. 